\title{
Health economic evaluation in orthotics and prosthetics: a systematic review protocol
}

\author{
Leigh Clarke ${ }^{1,2^{*}} \mathbb{D}$, Michael Dillon ${ }^{1}$ and Alan Shiell ${ }^{3}$
}

\begin{abstract}
Background: Health economic evaluations are essential to support health care policy and investment decisions. To date, health economic evaluations in orthotics and prosthetics have focused on discrete components of an orthosis/prosthesis (e.g. a microprocessor controlled prosthetic knee joint) rather than the broader service provided by orthotist/prosthetists. As such, the contribution to orthotic/prosthetic policy and investment decisions is unclear. Whilst there are opportunities to conduct more informative health economic evaluations that describe the costs and benefits of the orthotic/prosthetic service, it is important that prospective research is informed by a critical review of the method design challenges and an understanding of how this research can be improved. The aim of this systematic review is to critically appraise the existing orthotic/prosthetic health economic evaluation literature and therefore determine evidence gaps, critical method design issues and the extent to which the literature informs orthotic/prosthetic policy and investment decisions.

Methods: A comprehensive range of databases_AMED, EMBASE, MEDLINE and PsychINFO, Cumulative Index of Nursing and Allied Health Literature (CINAHL), ProQuest Nursing and Allied Health, Web of Science, Cochrane Database of Systematic Reviews (CDSR) and specialty health economic databases — will be searched using National Library of Medicine Medical Subject Headings (MeSH) terms as well as the title, abstract, and keyword terms. Search terms related to the intervention (e.g. orthosis), including variants used by varying professional disciplines (e.g. brace), will be used in preference to defining the populations that use orthotic and prosthetic services (e.g. people living with rheumatoid arthritis). Search terms related to health economic evaluations will be guided by previously developed and tested search strings and align with recommendations by the Canadian Agency for Drugs and Technologies in Health. Articles meeting the inclusion criteria will be hand-searched for relevant citations, and a forward citation search using Google Scholar will also be conducted to identify early online articles not yet indexed in traditional databases. Original research published in the English language and after 1 January 2000 will be included. The Checklist for Health Economic Evaluation Reporting Standards (CHEERS) and the Consensus on Health Economic Criteria (CHEC)-Extended list will be used to appraise the methodological quality and identify sources of bias. Data extraction and appraisal will be conducted by one reviewer independently using appraisal instrument guidelines and a content specific decision aid with exemplars. A subsequent review by a second researcher will be undertaken to confirm the accuracy of the extraction and appraisal, and a final review by a third where consensus cannot be reached. The data will be extracted to a purpose-built data extraction template with decision-making guidelines to support consistency. Where possible, the findings of the review will be reported as a meta-analysis, although the heterogeneity of the literature will likely mean a narrative review that illuminates method design issues that contribute to imprecision and variation will be more appropriate.

(Continued on next page)
\end{abstract}

\footnotetext{
* Correspondence: leigh.clarke@aopa.org.au

'Discipline of Prosthetics and Orthotics, Department of Physiotherapy,

Podiatry, Prosthetics and Orthotics, School of Allied Health, Human Services

and Sport, La Trobe University, Melbourne, Victoria 3083, Australia

${ }^{2}$ The Australian Orthotic Prosthetic Association Ltd, Melbourne, Victoria 3124,

Australia

Full list of author information is available at the end of the article
}

(C) The Author(s). 2019 Open Access This article is distributed under the terms of the Creative Commons Attribution 4.0 International License (http://creativecommons.org/licenses/by/4.0/), which permits unrestricted use, distribution, and reproduction in any medium, provided you give appropriate credit to the original author(s) and the source, provide a link to the Creative Commons license, and indicate if changes were made. The Creative Commons Public Domain Dedication waiver (http://creativecommons.org/publicdomain/zero/1.0/) applies to the data made available in this article, unless otherwise stated. 
(Continued from previous page)

Discussion: This protocol has been purposefully designed to summarise the existing evidence and appraise the methodological approaches used and the quality of the health economic evaluations in orthotics and prosthetics. What we learn from this review will be used to guide further work in this area and design more rigorous health economic evaluations into the future.

Systematic review registration: PROSPERO CRD42018116910.

Keywords: Systematic review, Economic evaluation, Health economics, Cost-effectiveness, Cost-benefit, Orthotics, Prosthetics

\section{Background}

People with mobility-related impairments, such as stroke, cerebral palsy or amputation, use orthoses/prostheses to assist with function, activity and participation [1].

Historically, orthoses/prostheses were provided by highly skilled tradespeople with little responsibility beyond the manufacture and supply of the orthosis/prosthesis [2-4], and as such, there was significant medical oversight for the associated clinical role which included prescription and reviews [2].

Over time, the role of the orthotist/prosthetist has become increasingly clinical $[2,5]$ as education and training have shifted from an apprenticeship model to tertiary education that emphasised the clinical role necessary for autonomous professional practice [3]. As such, many aspects of the clinical role that were once provided by medical specialists are now the responsibility of the orthotist/prosthetist including prescription, reviews and education, as illustrative examples $[3,5]$.

Into the future, the clinical role of the orthotist/prosthetist will continue to evolve with the introduction of new, contemporary models of care that focus on supporting clients to identify the goals of their treatment [6] and measure how successfully these goals have been achieved. By way of example, the Australian National Disability Insurance Scheme, a national funding model for disability services and interventions, has been designed to redress the historical, paternalistic prescribing role of practitioners [7]. Instead, practitioners are expected to use high-level communication skills to facilitate discussions, thereby supporting clients to identify their own treatment goals $[6,8]$ and inform their decision about orthotic/prosthetic interventions given an understanding of the likely outcomes [7, 9]. Practitioners are also expected to evaluate the effectiveness of an intervention using valid and reliable outcome measures $[10,11]$ and, in this way, demonstrate the contribution that the intervention makes towards the attainment of a client's goals.

The enhanced clinical role of the orthotist/prosthetist and the introduction of more contemporary models of care have fundamentally changed the way orthotic/prosthetic clinical services are provided. However, the small pool of orthotic/ prosthetic health economic evaluation literature has remained focussed on evaluating the cost-effectiveness of newer, high- cost technologies (e.g. microprocessor prosthetic knees or bone-anchored prostheses) compared to the current standard technologies [12-17]. Whilst these studies make a meaningful contribution to technology-related policy decisions, they do not inform decisions regarding the funding of comprehensive clinical services provided by orthotist/prosthetists that focus on supporting clients to achieve their goals of which the provision of technology is a component.

A prudent first step to developing a contemporary approach to orthotic and prosthetic health economic evaluations would be to conduct a systematic review of the current orthotic/prosthetic literature. Critical appraisal of the available health economic evaluation research would identify evidence gaps and critical method design issues that could inform an assessment of the extent to which current literature can meaningfully contribute to decisions about funding of contemporary clinical services of which provision of an orthosis/prosthesis is part. The outcomes of the systematic review could also inform the design of more rigorous health economic evaluations into the future.

In summary, health economic evaluations are essential to support health care policy and investment decisions. To date, health economic evaluations in orthotics and prosthetics have focused on discrete components of an orthosis/prosthesis (e.g. a microprocessor-controlled prosthetic knee joint), and whilst these studies can help inform technology-related policy decisions, they are unlikely to be well-designed to inform decisions about contemporary clinical services in which technologies, such as prostheses and orthoses, are provided. Therefore, it is unclear whether current evaluations adequately contribute to costeffectiveness knowledge, policy and investment decisions for orthotic/prosthetic services. Into the future, there are opportunities to conduct health economic evaluations where the costs and benefits of the orthotic/prosthetic service are measured in contemporary healthcare models, such as the National Disability Insurance Scheme, therefore informing health care policy and investment decisions for people requiring orthoses/prostheses within such schemes. A prudent first step would be to conduct a systematic review of the current orthotic/prosthetic health economic literature to identify evidence gaps, and method design issues that can inform the design of rigorous health economic 
evaluations focused on the cost-effectiveness of contemporary services of which the provision of a prosthesis/orthosis is part.

The aim of this systematic review is to critically appraise the existing orthotic/prosthetic health economic evaluation literature and therefore determine evidence gaps, critical method design issues and the extent to which the literature informs orthotic/prosthetic policy and investment decisions.

\section{Method}

\section{Search strategy}

The OVID platform will be used to search the following databases independently: AMED, EMBASE, MEDLINE and PsychINFO. Separate searches will also be conducted for Cumulative Index of Nursing and Allied Health Literature (CINAHL), ProQuest Nursing and Allied Health, Web of Science and the Cochrane Database of Systematic Reviews (CDSR). In line with recent search strategy recommendations, core database searches will be complemented with health economic specific database searches including Centre for Reviews and Dissemination (CRD) Health Technology Assessment and the National Health Service Economic Evaluation Database (NHS EED) [18-21].

National Library of Medicine Medical Subject Headings $(\mathrm{MeSH})$ will be used for the relevant aforementioned databases, including the terms for Artificial Limbs, Amputation, Amputees, Orthotic Device, Costbenefit Analysis, Costs and Cost Analyses and Qualityadjusted Life Years. These MeSH terms include all relevant root and hierarchical branches related to the intervention (i.e. prostheses and orthoses) and outcome (e.g. health economic evaluation, cost-effectiveness) and will also be exploded to reduce the likelihood that relevant publications will be overlooked due to variations in the way articles are indexed.

Search terms related to the intervention (i.e. prostheses and orthoses), as well as variants used by varying professional disciplines (e.g. splint, brace), will be used in preference to defining the populations that use orthotic and prosthetic services which are very broad (e.g. cerebral palsy, stroke, scoliosis, rheumatoid arthritis, plagiocephaly). Search terms related to health economic evaluations (e.g. cost-effectiveness, cost-utility) will be based on previously developed and tested search strings [22, 23] and align with recommendations by the Canadian Agency for Drugs and Technologies in Health [20].

Synonyms and acronyms for each of the aforementioned search terms will be used in combination with Boolean operators and wild cards to ensure variations in spelling and punctuation (e.g. cost effectiveness and cost-effectiveness) are captured. Search terms and larger search strings will be developed, tested and modified based on the search results from each database that highlight terms in the title (ti), abstract (ab) and keyword (kw) that were relevant. Where these field codes vary across databases, the appropriate alternative will be used.

Search terms designed to exclude irrelevant literature will also be used given a large body of research relating to internal or dental prostheses (e.g. hip or dental implants). In this way, studies pertaining to internal hip and knee replacements and other non-limb prosthetic results (e.g. aortic, valve, breast) will be excluded. It is known that dental literature uses numerous terms (e.g. overdentures, implant-supported, or restoration) that are also non-dental specific (e.g. implant) therefore preventing efficient exclusion using single search terms and will inadvertently exclude relevant prosthetic literature (e.g. osseointegration studies in which the term implant is used). Therefore, instead of using search terms to exclude irrelevant dental literature, a MeSH search strategy will be used (i.e. Exp Dentistry/), allowing exclusion of all studies indexed under this subject heading, including all root and hierarchical branches.

The aforementioned MeSH and search terms were developed through an analysis of the keywords, titles and abstracts of known relevant journal articles. As part of protocol development, and to ensure the rigour of this approach, testing was undertaken for one database. This included an assessment of the comprehensiveness and precision of the search yield against a pool of known orthotic and prosthetic health economic evaluation studies with wide-ranging publication years, keywords and journals. Where the search failed to identify known articles, the search terms and strings were refined and retested. The testing also allowed for confirmation of search methodology decisions, for example, the use of intervention only search term (i.e. exclusion of population terms) was found to be sufficient to identify a pool of known articles without defining the patient population and generic search terms related to quality of life (QoL) yielded substantial literature of a general nature, unrelated to health economic evaluation in testing, therefore confirming the reasonableness of its exclusion.

Given that the author's native language is English and that restriction of non-English language articles does not alter the conclusions of systematic reviews and meta-analyses [24], all search results will be limited to studies published in the English language. The search results will also be limited by time (i.e. 1 January 2000 to end search date). Time limits were tested as part of search strategy development. We were unable to identify any orthotic and prosthetic literature that met the definition of a health economic evaluation published prior to the year 2000. This is due to the introduction of health technology assessment measures in the late 1990s (e.g. the National Institute for Health and Care Excellence (NICE) in the UK was established in 1999), which provided guidance for contemporary methodological approaches in the evaluation of new healthcare technologies, and therefore 
stimulated health economic evaluation research with a reported increase from 2007 to 2012 of $79 \%$ and $108 \%$ in the NHS Economic Evaluation Database (NHS HEED) and PubMed, respectively [21].

The search results will also be limited to original, peerreviewed research and as such, commentaries and reviews will be excluded. As part of protocol development, the decision to exclude grey literature was tested. We identified grey literature that met the operational definition of a health economic evaluation; however, they were concurrently published in peer-review journals and, as such, the journal publication provided a stronger form of evidence (e.g. Dobson DaVanzo \& Associates Report 2013 and the Queensland Artificial Limb Scheme Report 2018 are also peer-review published) [14, 25-27]. This testing was conducted through hand-searching the reference list of known peer-reviewed studies $[12-17,28-30]$ and an assessment of known grey literature $[25,27,31,32]$ and confirmed the appropriateness of this search strategy limitation.

The reference lists of articles that meet the inclusion criteria will be hand-searched. A forward citation search will be conducted using Google Scholar as this platform does do not rely on traditional indexation methods and is therefore better able to identify early online publications.

As required by the Preferred Reporting Items for Systematic Reviews and Meta-Analyses Protocol (PRISMAP) guidelines [33], an illustrative search is documented for one database (Table 1).

\section{Data management}

The search results will be exported to EndNote X8.2 (Thomson Reuters Inc.). Duplicates will be removed using the EndNote "find duplicates" feature and through hand searching given variation in spelling or punctuation often prevent automatic detection of duplicates. Fulltext articles will be appended to each EndNote record for studies that meet the inclusion criteria or where fulltext articles are required to determine eligibility.

All EndNote records will be exported to a Microsoft Excel spreadsheet using a custom-made Endnote output style capturing key bibliographic information (e.g. authors, year, journal title). The Excel file will be customised to allow for the recording of decisions regarding inclusion/exclusion, data extraction and recording the checklist items from the critical appraisal of each article. A separate tab will also be used to record the results of screening based, so as to populate the PRISMA search flowchart (Additional file 1).

\section{Selection process}

Articles will be included if they met the following criteria:

1. Research type: original, peer-reviewed research;
2. Language: published in the English language;

3. Year of publication: published since 1 January 2000;

4. Intervention: externally applied orthoses or prostheses for any part of the human body as defined by the International Standards, ISO 9999:2016 (ISO 8549 1-3 prosthetics and orthotics - vocabulary) [34], and provided by a healthcare profession as defined in the Health Practitioner Regulation National Law Act 2009 [35] and regulated via the Australian Health Practitioner Regulation Agency (AHPRA) in Australia or recognised by the National Alliance of Self Regulating Health Professions (NASRHP) (https:// nasrhp.org.au/);

5. Outcome: a health economic evaluation, defined as "the comparative analysis of alternative courses of action in terms of both their costs and consequences" [18].

The operational definition of orthoses or prostheses (i.e. the intervention) will exclude studies of internal devices (e.g. hip and knee replacements or heart valve replacements). Where there are simultaneous treatments forming a cointervention, such that the study does not inform the costeffectiveness of a discrete orthotic/prosthetic device or service, the study will be excluded (i.e. conservative management versus surgical management of repetitive strain injuries, where the conservative intervention is multi-faceted including combinations of bracing and physiotherapy). The intervention under investigation must be provided by a recognised health care profession as defined by AHPRA or NASRHP, in which orthotic and/or prosthetic services are accepted as within the practitioner's scope of practice. This definition acknowledges the substantial overlap in the scope of practice for several health professions (e.g. occupational therapists and orthotists both provide upper limb orthotic services, and podiatrists and orthotists both provide foot orthotic services). The operational definition of health economic evaluation will limit the inclusion of studies to those using cost-effectiveness, cost-utility, cost-benefit, costminimisation or studies with multiple outcome measures (cost-consequence) methodologies, either as experimental trials or through simulation (i.e. a Markov model) $[18,20]$. As such, studies describing the cost of the intervention (i.e. cost comparison studies) will be excluded.

Due to the unambiguous nature of the inclusion criteria which does not require complex judgement, the search yield will be screened by one investigator (LC) based on a review of the title and abstract [36]. Full-text articles will be retrieved and reviewed as necessary to determine inclusion. A second opinion from another investigator (MD) will be sought as required and any disagreement will be resolved through discussion until consensus. Where consensus cannot be reached, a third 
Table 1 Example search for MEDLINE database to identify health economic evaluation literature in orthotics and/or prosthetics

\begin{tabular}{|c|c|c|c|}
\hline $\begin{array}{l}\text { Search } \\
\text { number }\end{array}$ & Topic & $\begin{array}{l}\text { Search } \\
\text { field }\end{array}$ & Search term \\
\hline 1 & & $\mathrm{MeSH}$ & Exp Artificial Limbs/ \\
\hline 2 & & $\mathrm{MeSH}$ & Exp Amputation/ \\
\hline 3 & & $\mathrm{MeSH}$ & Exp Amputees/ \\
\hline 4 & & $\mathrm{MeSH}$ & Exp Orthotic Devices/ \\
\hline 5 & & $\mathrm{MeSH}$ & Exp Cost-benefit Analysis/ \\
\hline 6 & & MeSH & Exp Costs and Cost Analysis/ \\
\hline 7 & & $\mathrm{MeSH}$ & Exp Economic Evaluation/ \\
\hline 8 & & $\mathrm{MeSH}$ & Exp Quality-adjusted life years/ \\
\hline 9 & & $\mathrm{MeSH}$ & Exp Dentistry/ \\
\hline 10 & & & $(\mathrm{OR} / 1-8)$ NOT 9 \\
\hline 11 & Intervention & $\begin{array}{l}\text { ti, ab, } \\
\text { kw }\end{array}$ & prosthe* OR orthot* OR orthos\#s OR splint* OR brace* OR bracing \\
\hline 12 & $\begin{array}{l}\text { Outcome-general health } \\
\text { economic terms }\end{array}$ & $\begin{array}{l}\text { ti, ab, } \\
\text { kw }\end{array}$ & $\begin{array}{l}\text { ((economic ADJ1 (impact OR value OR factor* OR analysis OR cost OR evaluation*)) OR (cost* } \\
\text { ADJ1 (health care OR hospital OR medical))) }\end{array}$ \\
\hline 13 & $\begin{array}{l}\text { Outcome-health economic } \\
\text { evaluation type }\end{array}$ & $\begin{array}{l}\text { ti, ab, } \\
\text { kw }\end{array}$ & $\begin{array}{l}\text { (cost* ADJ1 (util* OR effective* OR efficacy* OR effic* OR benefit* OR consequence* OR analy* } \\
\text { OR minimi* OR comparison OR saving* OR breakdown OR lowering OR estimate* OR variable* } \\
\text { or allocation OR control)) }\end{array}$ \\
\hline 14 & $\begin{array}{l}\text { Outcome-health economic } \\
\text { measure used }\end{array}$ & $\begin{array}{l}\text { ti, ab, } \\
\text { kw }\end{array}$ & $\begin{array}{l}\text { (((value OR values OR valuation) ADJ2 (money OR monetary OR life OR lives OR costs OR cost)) } \\
\text { OR (quality-adjusted life ADJ1 (year* OR expectanc*)) OR (quality adjusted life ADJ1 (year* OR } \\
\text { expectanc*)) OR (QOLY* OR QALY* OR QALE*)) }\end{array}$ \\
\hline 15 & & & 10 AND 11 AND (OR/12-14) \\
\hline 16 & Exclusion & ti & $\begin{array}{l}\text { [15] NOT (breast OR fracture* OR arthrop* OR angiop* OR transplant* OR heart OR aort* OR } \\
\text { valve* OR stent* OR retina* OR ocular OR hernia OR erect* OR carcinoma OR disc OR (total OR } \\
\text { replacement* ADJ2 (hip OR knee OR ankle OR joint OR vascular)) OR (implant* ADJ2 cochlear) } \\
\text { OR (cost ADJ3 (walking OR energy OR metabolic))) }\end{array}$ \\
\hline 17 & & & Limit 16 to English language \\
\hline
\end{tabular}

Field codes: MeSH National Library of Medicine Subject Headings, Exp exploded, ti title, $a b$ abstract, kw keyword as identified by author(s)

reviewer (AS) will be called upon to provide advice and resolve conflict as necessary.

\section{Quality appraisal and risk of bias}

The Consolidated Health Economic Evaluation Reporting Standards (CHEERS) checklist [18] will be used to appraise quality of the reporting. In concert, the Consensus on Health Economic Criteria (CHEC)-Extended list [37, 38] will be used to assess risk of bias of individual studies.

The CHEERS checklist was developed by the International Society for Pharmacoeconomics and Outcomes Research (ISPOR) Health Economic Evaluation Publication Guidelines Good Reporting Practices Task Force to facilitate consistent and transparent reporting of economic evaluation research and represents the most upto-date and accepted standards in this area [18]. Whilst several researchers have used the CHEERS checklist as a risk of bias appraisal tool, its purpose is to guide researchers, editors and peer reviewers in best practice reporting for health economic evaluations and was not intended to assess the quality of the method design or execution of the study [18].
The CHEC-Extended list is recommended for the assessment of risk of bias where the literature contains both model-based and clinical trial or observational studies [39], as is the case in orthotics and prosthetics literature. The CHEC-Extended list is supported by detailed guidelines outlining the value judgement for each criterion and would likely address the poor inter-rater agreement inherent in the original CHEC list (0.33 intraclass correlation co-efficient; 95\% CI 0.07-0.71) [40].

Whilst both the CHEC-Extended list and the CHEERS checklist have a small number of criteria that overlap (e.g. the description of the study population, description of competing alternatives and the definition of the research question), they will be used in their entirety, as originally designed and endorsed through a Delphi consensus process [38].

\section{Data extraction and risk of bias assessment process}

A data extraction spreadsheet (Additional file 2), developed in Microsoft Excel, will be used to systematically record details of the intervention and setting (e.g. target population, type of intervention, setting/data source), characteristics of the sample (e.g. age, sex, time since amputation/diagnosis), features of the 
method design (e.g. type of health economic evaluation, time horizon, perspective, discounting methods and structural assumptions) and the recording of results (e.g. benefit outcome, cost outcome, and the incremental cost-effectiveness ratio). This approach has been described in comparable health economic evaluation systematic reviews [37].

The spreadsheet will include fields as specified in the CHEERS checklist and the CHEC-Extended list to allow for systematic recording of the appraisal outcomes. To reduce errors and omissions, the spreadsheet will be intuitively set up. For example, detailed questions, decision rules and guidelines are available by "scrolling-over" the column heading. Pull down menus with standard responses will be used to aid consistent data entry. Commentary space will be available for both the CHEERS checklist and CHEC-Extended list to record notes and justify decisions within pre-determined headings (e.g. subjects, methods, assumptions).

As part of protocol development, the data extraction spreadsheet has been developed and tested on two studies from the known bank of orthotic and prosthetic health economic evaluation studies. During testing, it became evident that the CHEC-Extended list guidelines could be used more reliably with a supporting content-specific decision aid that provides examples to contextualise the guidelines relative to the specific area of healthcare under review (Additional file 3).

Data extraction and completion of the CHEERS and CHEC-Extended will be undertaken by a primary reviewer (LC). A second reviewer (MD) will confirm the accuracy of these data and the risk of bias and reporting assessment and disagreement resolved through discussion. Single data extraction results in substantial time savings and is reported to have similar error rates to duplicate data extraction and appraisal, with minimal impact on the final extraction outcome $[41,42]$. Furthermore, the appraisal tools have published decision guidelines which are further supported by our context-specific decision aid with exemplars, which reduce the likelihood of error. As necessary, a third reviewer (AS) will be called upon to provide advice or resolve conflict using discussion until consensus. Where necessary, the authors of the original research will be contacted to clarify aspects of the method design, result and/or reporting.

\section{Data summary and reporting}

Initial scoping of the literature indicated that there are few health orthotic/prosthetic economic evaluations, and those identified use a diverse range of study designs, outcome measures and patient populations. Whilst we anticipate this will make a meta-analysis inappropriate, this will be tested through the completion of the data extraction process and where feasible a meta-analysis will be conducted. Where meta-analysis is not feasible, then the findings from this review will be reported as a narrative. The narrative will outline common issues with the reporting and method design that introduce bias with a view to explain the underlying cause of heterogeneity and imprecision that reduce confidence in the results and limit the synthesis of outcomes across studies. A discussion of the extent to which the literature contributes to orthotic and prosthetic policy and investment decisions will also be provided.

\section{Discussion}

To our knowledge, this is the first systematic review of health economic evaluations pertaining to all orthotic and prosthetic interventions and services.

This protocol has been purposefully designed to summarise the existing evidence and, in doing so, appraise the methodological approaches used and the quality of the health economic evaluations relating to orthotics and prosthetics. What we learn from this review will be used to guide further work in this area and design more rigorous health economic evaluations into the future.

\section{Additional files}

Additional file 1: PRISMA-P checklist (DOCX $17 \mathrm{~kb}$ )

Additional file 2: Data extraction template (XLSX 56 kb)

Additional file 3: CHEC-Extended content-specific decision aid (orthotics and prosthetics) (DOCX $121 \mathrm{~kb})$

\section{Abbreviations}

CDSR: Cochrane Database of Systematic Reviews; CHEC: Consensus on Health Economic Criteria list; CHEERS: Consolidated Health Economic Evaluation Reporting Standards; CINAHL: Cumulative Index of Nursing and Allied Health Literature; CRD: Centre for Reviews and Dissemination; HEED: Health economic evaluations database; MeSH: Medicine medical subject heading; NHS: National Health Service; NICE: National Institute for Health and Care Excellence; PRISMA: Preferred Reporting Items for Systematic Reviews and Meta-Analyses; PRISMA-P: Preferred Reporting Items for Systematic Reviews and Meta-Analyses Protocol

\section{Acknowledgements}

Not applicable

\section{Authors' contributions}

LC led the development and writing of the protocol. MD and AS provided critical review. LC and MD led the development of the justification; established the aims; developed, tested and revised the search strategy; and developed the procedures for assessment of risk of bias. AS provided critical review and health economic expertise. LC developed, tested and revised the data extraction template and appraisal decision aid. MD tested the extraction template, reviewed the appraisal decision aid and provided feedback. LC developed the procedures for data management and data reporting and MD provided feedback. LC and MD drafted the discussion. All authors reviewed the protocol for important intellectual content. The guarantor of the review is LC. All authors have read and approved the final manuscript.

Authors' information

Not applicable

\section{Funding}

LC was provided with study leave support from The Australian Orthotic Prosthetic Association to conduct this work. The supporter did not have any role in the design of the protocol, writing of the manuscript or decision to submit for publication. 


\section{Availability of data and materials}

Not applicable

\section{Ethics approval and consent to participate \\ Not applicable}

\section{Consent for publication}

Not applicable

\section{Competing interests}

The authors declare that they have no competing interests.

\section{Author details}

'Discipline of Prosthetics and Orthotics, Department of Physiotherapy, Podiatry, Prosthetics and Orthotics, School of Allied Health, Human Services and Sport, La Trobe University, Melbourne, Victoria 3083, Australia. ${ }^{2}$ The Australian Orthotic Prosthetic Association Ltd, Melbourne, Victoria 3124 Australia. ${ }^{3}$ Department of Public Health, School of Psychology and Public Health, La Trobe University, Melbourne, Victoria 3083, Australia.

Received: 8 December 2018 Accepted: 5 June 2019

Published online: 27 June 2019

\section{References}

1. World Health Organization. International classification of impairments, disabilities, and handicaps: a manual of classification relating to the consequences of disease published in accordance with resolution WHA29.35 of the twenty-ninth world health assembly. Geneva: World Health Organization; 1980.

2. Blocka D. Moving the profession. Prosthetics Orthot Int. 2008;32(3):282-6.

3. Hovorka CF, Shurr DG, Bozik DS. The concept of an entry-level interdisciplinary graduate degree preparing orthotists for the new millennium part 1: history of orthotic and prosthetic education. J Prosthet Orthot. 2002;14(2):51-8.

4. Radford DJ. Grey coats or white coats?: the emergence of clinical prosthetists in Australia: La Trobe University; 1993. Available from: https:// books.google.com.au/books?id=v9RvNQAACAAJ.

5. Ash S, O'Connor J, Anderson S, Ridgewell E, Clarke L. A mixed-methods research approach to the review of competency standards for orthotist/ prosthetists in Australia. Int J Evid Based Healthc. 2015;13(2):93-103.

6. Brown M. Participation: the insider's perspective. Arch Phys Med Rehabil. 2010;91(9 Suppl):S34-7.

7. Crozier M, Muenchberger H, Colley J, Ehrlich C. The disability self-direction movement: considering the benefits and challenges for an Australian response. Aust J Soc Issues. 2013:48(4):455-72.

8. Roepke AM, Williams RM, Turner AP, Henderson AW, Norvell DC, Henson $H$, et al. A longitudinal study of social participation after dysvascular lower extremity amputation. Am J Phys Med Rehabil. 2017;96(10):741-7.

9. Sherwood A, Brinkmann J, Fatone S. Review of benefits to practitioners of using good patient-practitioner communication. JPO: J Prosthet Orthot. 2018;30(1):5-12.

10. Condie E, Scott H, Treweek S. Lower limb prosthetic outcome measures: a review of the literature 1995 to 2005. J Prosthet Orthot. 2006;18(6):P13-45.

11. Gaunaurd I, Spaulding SE, Amtmann D, Salem R, Gailey R, Morgan SJ, et al Use of and confidence in administering outcome measures among clinical prosthetists: results from a national survey and mixed-methods training program. Prosthetics Orthot Int. 2015;39(4):314-21.

12. Brodtkorb TH, Henriksson M, Johannesen-Munk K, Thidell F. Costeffectiveness of C-leg compared with non-microprocessor-controlled knees: a modeling approach. Arch Phys Med Rehabil. 2008;89(1):24-30.

13. Cutti AG, Lettieri E, Del Maestro M, Radaelli G, Luchetti M, Verni G, et al. Stratified cost-utility analysis of C-leg versus mechanical knees: findings from an Italian sample of transfemoral amputees. Prosthetics Orthot Int. 2017:41(3):227-36

14. Frossard LA, Merlo G, Burkett B, Quincey T, Berg D. Cost-effectiveness of bone-anchored prostheses using osseointegrated fixation: myth or reality? Prosthetics Orthot Int. 2018;42(3):318-27.

15. Gerzeli S, Torbica A, Fattore G. Cost utility analysis of knee prosthesis with complete microprocessor control (C-leg) compared with mechanical technology in trans-femoral amputees. Eur J Health Econ. 2009;10(1):47-55.

16. Hansson E, Hagberg K, Cawson M, Brodtkorb TH. Patients with unilateral transfemoral amputation treated with a percutaneous osseointegrated prosthesis: a cost-effectiveness analysis. Bone Joint J. 2018;100-B(4):527-34.
17. Seelen H, Hemmen B, Schmeets AJ, Ament AJHA, Evers S. Costs and consequences of a prosthesis with an electronically stance and swing phase controlled knee joint. Technol Disabil. 2009;21:25-34.

18. Husereau D, Drummond M, Petrou S, Carswell C, Moher D, Greenberg D, Augustovski F, Briggs AH, Mauskopf J, Loder E. Consolidated Health Economic Evaluation Reporting Standards (CHEERS) statement. BMJ. 2013;346.

19. Thielen FW, Van Mastrigt G, Burgers LT, Bramer WM, Majoie H, Evers S, et al. How to prepare a systematic review of economic evaluations for clinical practice guidelines: database selection and search strategy development (part 2/3). Expert Rev Pharmacoecon Outcomes Res. 2016;16(6):705-21.

20. Glanville J, Fleetwood K, Yellowlees A, Kaunelis D, Mensinkai S. Development and testing of search filters to identify economic evaluations in MEDLINE and EMBASE. Ottawa: Canadian Agency for Drugs and Technologies in Health; 2009

21. Wood H, Arber M, Glanville JM. Sytematic review of economic evaluations: how extensive are their searches? Int J Technol Assess Health Care. 2017;33(1):25-31.

22. Howard-Wilsher S, Irvine L, Fan H, Shakespeare T, Suhrcke M, Horton S, et al. Systematic overview of economic evaluations of health-related rehabilitation. Disabil Health J. 2016;9(1):11-25.

23. Sassi F, Archard L, McDaid D. Searching literature databases for health care economic evaluations: how systematic can we afford to be? Med Care. 2002;40(5):387-94

24. Morrison A, Polisena J, Husereau D, Moulton $\mathrm{K}$, Clark M, Fiander M, et al. The effect of English-language restriction on systematic review-based metaanalyses: a systematic review of empirical studies. Int J Technol Assess Health Care. 2012;28(2):138-44.

25. Dobson DaVanzo \& Associates. Retrospective cohort study of the economic value of orthotic and prosthetic services among medicare beneficiaries. Vienna, VA: Dobson DaVanzo \& Associates; 2013

26. Dobson A, El-Gamil A, Shimer M, DaVanzo JE. Economic value of prosthetic services among Medicare beneficiaries: a claims-based retrospective cohort study. Mil Med. 2016;181(2 Suppl):18-24.

27. Berg D, Frossard LA, Burkett B. Innovations of health services and economic evaluation of bone-anchored prosthesis using osseointegration: The Queensland Artificial Limb Service's experience. Brisbane, Queensland: YourResearch Project; 2018.

28. Fatoye $F$, Haigh $C$. The cost-effectiveness of semi-rigid ankle brace to facilitate return to work following first-time acute ankle sprains. J Clin Nurs. 2016;25(9-10):1435-43.

29. Janssen KW, Hendriks MR, van Mechelen W, Verhagen $\mathrm{E}$. The costeffectiveness of measures to prevent recurrent ankle sprains: results of a 3arm randomized controlled trial. Am J Sports Med. 2014;42(7):1534-41.

30. Rome K, Clark H, Gray J, McMeekin P, Plant M, Dixon J. Clinical effectiveness and cost-effectiveness of foot orthoses for people with established rheumatoid arthritis: an exploratory clinical trial. Scand J Rheumatol. 2017;46(3):187-93.

31. Three Rivers Consulting. Enable NSW Prosthetic Limb Funding Model Review: Final report. Sydney, NSW: three Rivers consulting; 2011.

32. Layton $\mathrm{N}$, Irlam C. Assistive technology for older Australians: rapid evidence review and economic pathways analysis. Canberra, ACT: National Aged Care Alliance; 2018.

33. Moher D, Liberati A, Tetzlaff J, Altman DG. Preferred reporting items for systematic reviews and meta-analyses: the PRISMA statement. PLoS Med. 2009;6(7):e1000097.

34. International Organization for Standardization (ISO). Assistive products for persons with disability -- classification and terminology (ISO 9999), ISO/TC 173/SC 2. Geneva, Switzerland: International Organization for Standardization (ISO; 2016.

35. Office of the Queensland Parliamentary Council. Health Practitioner Regulation National Law Act 2009. Queensland, Australia 2010.

36. Cooper H, Ribble RG. Influences on the outcome of literature searches for integrative research reviews. Knowledge. 1989;10(3):179-201.

37. Odnoletkova I, Goderis G, Pil L, Nobels F, Aertgeerts B, Annemans L, et al. Cost-effectiveness of therapeutic education to prevent the Development and progression of type 2 diabetes. Systematic review. J Diabetes Metab. 2014:5(9) Appendix

38. Evers $\mathrm{S}$, Goossens M, de Vet $\mathrm{H}$, van Tulder M, Ament A. Criteria list for assessment of methodological quality of economic evaluations: consensus on health economic criteria. Int J Technol Assess Health Care. 2005;21 (2):240-5.

39. van Mastrig G, Hiligsmann M, Arts J, Broos P, Kleijnen J, Evers S, et al. How to prepare a systematic review of economic evaluations for informing evidence-based healthcare decisions: a five-step approach (part 1/3). Expert Rev Pharmacoecon Outcomes Res. 2016;16(6):689-704. 
40. Gerkens S, Crott R, Cleemput I, Thissen JP, Closon MC, Horsmans Y, et al. Comparison of three instruments assessing the quality of economic evaluations: a practical exercise on economic evaluations of the surgical treatment of obesity. Int J Technol Assess Health Care. 2008;24(3):318-25.

41. Jones AP, Remmington T, Williamson PR, Ashby D, Smyth RL. High prevalence but low impact of data extraction and reporting errors were found in Cochrane systematic reviews. J Clin Epidemiol. 2005;58(7):741-2.

42. Buscemi N, Hartling L, Vandermeer B, Tjosvold L, Klassen TP. Single data extraction generated more errors than double data extraction in systematic reviews. J Clin Epidemiol. 2006;59(7):697-703.

\section{Publisher's Note}

Springer Nature remains neutral with regard to jurisdictional claims in published maps and institutional affiliations.

Ready to submit your research? Choose BMC and benefit from:

- fast, convenient online submission

- thorough peer review by experienced researchers in your field

- rapid publication on acceptance

- support for research data, including large and complex data types

- gold Open Access which fosters wider collaboration and increased citations

- maximum visibility for your research: over $100 \mathrm{M}$ website views per year

At $\mathrm{BMC}$, research is always in progress.

Learn more biomedcentral.com/submissions 